\title{
Effect of Sb incorporation on the electronic structure of InAs quantum dots
}

\author{
A. G. Taboada, ${ }^{*}$ J. M. Llorens, D. Alonso-Álvarez, B. Alén, A. Rivera ${ }^{\dagger}$ Y. González, and J. M. Ripalda $^{\ddagger}$ \\ Instituto de Microelectrónica de Madrid, CNM (CSIC), C/Isaac Newton 8, PTM 28760 Tres Cantos, Madrid, Spain
}

(Received 6 March 2013; revised manuscript received 16 July 2013; published 8 August 2013)

\begin{abstract}
On the basis of optical characterization experiments and an eight band $k p$ model, we have studied the effect of $\mathrm{Sb}$ incorporation on the electronic structure of InAs quantum dots (QDs). We have found that Sb incorporation in InAs QDs shifts the hole wave function to the center of the QD from the edges of the QD where it is otherwise pinned down by the effects of shear stress. The observed changes in the ground-state energy cannot merely be explained by a composition change upon Sb exposure but can be accounted for when the change in lateral size is taken into consideration. The $\mathrm{Sb}$ distribution inside the QDs produces distinctive changes in the density of states, particularly, in the separation between excitation shells. We find a 50\% increase in the thermal escape activation energy compared with reference InAs quantum dots as well as an increment of the fundamental transition decay time with $\mathrm{Sb}$ incorporation. Furthermore, we find that $\mathrm{Sb}$ incorporation into quantum dots is strongly nonlinear with coverage, saturating at low doses. This suggests the existence of a solubility limit of the $\mathrm{Sb}$ incorporation into the quantum dots during growth.
\end{abstract}

DOI: 10.1103/PhysRevB.88.085308

PACS number(s): 78.67.Hc, 81.16.Dn, 78.55.Cr, 81.15.Hi

\section{INTRODUCTION}

New challenges in semiconductor research, such as photonic-based circuitry, high efficiency solar cells, or infrared detectors may find potential solutions in the application of the unique properties of self-assembled quantum dots (QDs). ${ }^{1-4}$ One of the most pursued research goals is the achievement of QD-based laser emission in the optical fiber telecommunications spectral window. Different groups have reached emission in the $1.55-\mu \mathrm{m}$ optical window with approaches based on heterostructures grown on GaAs substrates, ${ }^{5}$ and promising results were already obtained with the demonstration of laser emission around $1.3 \mu \mathrm{m} .{ }^{6,7}$ Despite this, realization of optically active QD layers emitting in the desired spectral range has no meaning for laser applications if the structures do not provide the benefits of low threshold current and high-temperature stability. To obtain these features two characteristics are required at room temperature (RT): full width at half maximum (FWHM) of the QD ensemble photoluminescence (PL) as small as possible (state of the art is less than $20 \mathrm{meV})^{8,9}$ and luminescence intensity as high as possible.

$\mathrm{Sb}$ compounds present ideal characteristics to redshift the QD emission wavelength and to increase the RT PL intensity due to the low band gap of antimonides and their characteristic low electron affinity. The introduction of strain-reducing capping layers of GaAsSb has been an intensively pursued approach. Akahane et al. and Liu et al. obtained InAs QD PL at $1.4 \mu \mathrm{m}$ by capping InAs QDs with GaAsSb. ${ }^{10,11} \mathrm{~A}$ similar approach was adopted by Ripalda et al., capping InGaAs QDs with GaAsSb. This capping layer leads to QD emission at $1.62 \mu \mathrm{m}$ at RT. ${ }^{12}$ This redshift was related with a type-I to type-II transition of the system energy levels with the hole deeply confined in the QD and the electron wave function delocalized in the capping quantum well (QW). Liu et al. have quantified the concentration of $\mathrm{Sb}$ that leads to such type-I to type-II transitions-14\% - in similar structures capped with a $\mathrm{GaAs}_{1-x} \mathrm{Sb}_{x}$ QW. ${ }^{13}$ More recently, Ulloa et al. have tuned the InAs QD emission energy between 1.15 and $1.50 \mu \mathrm{m}$ by modifying the $\mathrm{Sb}$ concentration $x$ of a 4.5-nm-thick $\mathrm{GaAs}_{1-x} \mathrm{Sb}_{x}$ QW grown as an InAs QD capping layer. ${ }^{14}$ Rapid thermal annealing of these samples resulted in type-II QDs with highly efficient luminescence which are, nevertheless, not optimal candidates as laser gain material due to the type-II alignment. $^{15}$

The $\mathrm{Sb}$ incorporation method on the InAs nanostructures plays a crucial role in InAsSb QDs' great performance for optical applications. Previous papers reporting on the optical characterization of InAsSb QDs directly grown on GaAs addressed that, independent of the Sb beam flux used during growth, the emission was blueshifted with respect to the InAs QD reference ensembles (blueshift ranging from 42 to $106 \mathrm{meV}){ }^{16}$ Further experiments carried out by this method achieved PL energy emission redshifts up to $50 \mathrm{meV}$. However, the broad size distribution characteristic of these InAsSb QD ensembles led to multimodal PL emission. ${ }^{17}$ In a previous paper, InAs QDs exposed to Sb before capping were presented as an alternative to enhance the RT luminescence in the 1.3- $\mu \mathrm{m}$ window. ${ }^{18}$ The optimized ensembles showed an order of magnitude increase in the PL intensity compared with the reference InAs/GaAs QDs with a FWHM of $18.5 \mathrm{meV}$ and an emission wavelength centered at $1.34 \mu \mathrm{m}$ at RT. These findings were discussed and were correlated with structural characterization-transmission electron microscopy (TEM) and cross-sectional scanning tunneling microscopy (X-STM) - which demonstrated Sb incorporation both in the nanostructures and in the capping layer.

In this paper, we analyze the effect of $\mathrm{Sb}$ incorporation on the electronic structure of these nanostructures in detail. PL experiments as a function of temperature, excitation energy, power density, as well as time-resolved PL (TRPL) characterization are presented. These results will be compared with an eight band $k p$ theoretical model based on the QDs' realistic dimensions and $\mathrm{Sb}$ distribution available from the mentioned structural characterization techniques. 
TABLE I. Summary of the different samples studied in this paper, their correspondent Sb concentration, and main emission energies. The values of the $\mathrm{Sb}$ concentration correspond to the top half of the QDs as reported in Ref. 18.

\begin{tabular}{lcccccc}
\hline \hline Sample & $\begin{array}{c}P_{\mathrm{Sb}} \\
(\mathrm{mbar})\end{array}$ & $\begin{array}{c}\text { Sb concentration } \\
(\%)\end{array}$ & $\begin{array}{c}\text { Lateral size } \\
(\mathrm{nm})\end{array}$ & $\begin{array}{c}\text { WL } \\
(\mathrm{eV})\end{array}$ & $\begin{array}{c}s \text { state } \\
(\mathrm{eV})\end{array}$ & $\begin{array}{c}s \text { state FWHM } \\
(\mathrm{meV})\end{array}$ \\
\hline $\mathrm{A}$ & $\mathrm{InAs} \mathrm{QD}$ & 0 & $15 \pm 3$ & 1.423 & 1.068 & 27.1 \\
$\mathrm{~B}$ & $2.0 \times 10^{-7}$ & $14.0 \pm 4.4$ & $15 \pm 3$ & 1.434 & 1.032 & 1.006 \\
$\mathrm{C}$ & $8.0 \times 10^{-7}$ & $11.6 \pm 3.5$ & $19 \pm 3$ & 1.434 & 1.6 \\
$\mathrm{D}$ & $3.1 \times 10^{-6}$ & $18.2 \pm 8.6$ & $20 \pm 3$ & 1.427 & 0.985 \\
\hline \hline
\end{tabular}

\section{METHOD}

Samples were grown by solid source molecular-beam epitaxy (MBE) on semi-insulating GaAs (001) substrates. QD nucleation was observed by reflection high-energy electron diffraction (RHEED) after deposition of 1.65 monolayers (MLs) of In at a $510{ }^{\circ} \mathrm{C}$ substrate temperature and at $0.02 \mathrm{ML} / \mathrm{s}$ In growth rate under $\mathrm{As}_{4}$ flux. Immediately after QD nucleation was detected, the substrate temperature was decreased to $440{ }^{\circ} \mathrm{C}$, whereas, In deposition continued up to a nominal thickness of 2.2 MLs. After InAs QD growth and before GaAs capping, the QDs were exposed to a Sb flux from a valved cracker $\mathrm{Sb}$ cell. The $\mathrm{Sb}$ exposure step had a duration of $10 \mathrm{~s}$ at beam equivalent pressures ranging from $2.0 \times 10^{-7}$ to $3.1 \times 10^{-6}$ mbar. In control samples, the $\mathrm{Sb}$ exposure step was substituted by an $\mathrm{As}_{4}$ exposure step at a beam equivalent pressure of $2.0 \times 10^{-6}$ mbar. In our MBE system, this value corresponds to an As incorporation rate of $1.5 \mathrm{ML} / \mathrm{s}$, calibrated by recording RHEED oscillations during group-V-limited $\mathrm{GaAs}$ growth. The GaAs capping temperature was $460{ }^{\circ} \mathrm{C}$ for the first $20 \mathrm{~nm}$, and then the temperature was ramped at $0.4{ }^{\circ} \mathrm{C} / \mathrm{s}$ up to $580{ }^{\circ} \mathrm{C}$ during a 5-min growth interruption. The capping was completed at this temperature up to a total thickness of $105 \mathrm{~nm}$. The Sb pressures corresponding to each sample are summarized in Table I. The notation presented in Table I will be used from now on.

The PL was investigated between 20 and $300 \mathrm{~K}$ for varying excitation power densities using a 532-nm frequency doubled Nd:YAG laser. The PL spectrum was recorded with a Peltier-cooled InGaAs photodiode array attached to a $0.3-\mathrm{m}$ focal length spectrograph. Excitation of the photoluminescence spectra (PLE) was measured at $16 \mathrm{~K}$ by passing the light of a halogen lamp through a monochromator with $0.5-\mathrm{nm}$ resolution. The PLE signal was collected with a reversed biased $\mathrm{Ge}$ detector after dispersion through a 0.3-m focal length monochromator. Time-resolved PL decay curves were measured at $20 \mathrm{~K}$ using a high-repetitionfrequency-picosecond-pulsed laser diode emitting at $405 \mathrm{~nm}$ as an excitation source. The emitted light was dispersed through a 0.3 -m focal length monochromator and was detected with an InGaAs near-infrared photomultiplier in the photoncounting mode. Overall time resolution of the system is $\sim 100$ ps after deconvolution of the instrument response function.

\section{RESULTS}

The different panels of Fig. 1 show normalized PL spectra obtained varying the excitation density at $T=20 \mathrm{~K}$ in samples grown under different Sb fluxes. As we discussed in our previous paper, antimony incorporation modifies the ground-state emission properties of the QDs as reflected in their PL peak energy, FWHM, and intensity. Compared with the reference InAs QD sample shown in Fig. 1(a), at the highest $\mathrm{Sb}$ dose, we are able to induce a maximum redshift of $83 \mathrm{meV}$ for the PL fundamental optical transition also reducing its FWHM from 27.1 to $22.7 \mathrm{meV}$. Up to a certain Sb dose, the RT PL intensity can be increased nearly one order of magnitude (sample $\mathrm{C}$ in Table II). In our samples, this maximum in PL intensity also occurs with the smallest FWHM (18.5 meV), and further exposure to $\mathrm{Sb}$ becomes detrimental in both aspects in good agreement with the morphological distortion previously reported for InAs QDs exposed to high Sb doses. ${ }^{18}$ Finally, the ground-state PL decay time $(\tau)$ measured at $T=20 \mathrm{~K}$ also changes with antimony incorporation increasing from $\tau_{\text {InAs }}=$ $0.98 \mathrm{~ns}$ in the reference sample to $\tau_{\text {sample D }}=1.53 \mathrm{~ns}$ at the highest $\mathrm{Sb}$ dose.

Antimony incorporation in the nanostructures is not only affecting the ground-state emission, but also affecting the excited shells electronic structure including the wetting layer (WL). Such changes are reflected in the PL excitation (PLE) spectra of each sample shown in Fig. 1. The GaAs edge is observed in all the ensembles at $E_{\mathrm{GaAs}}=1.521 \mathrm{eV}$ together with an acceptor level at $E=1.481 \mathrm{eV}{ }^{19}$ The reference InAs ensemble WL absorption edge is $1.423 \mathrm{eV}$. The introduction of a low-pressure $\mathrm{Sb}$ exposure step (samples $\mathrm{B}$ and $\mathrm{C}$ ) before GaAs capping results in a $12-\mathrm{meV}$ blueshift in the WL absorption resonance. We relate this initial blueshift with a narrowing of the WL due to the surfactant effect of $\mathrm{Sb}$ increasing the diffusion of In from the WL to the QDs and/or the evaporation of material due to the formation of more volatile $\mathrm{InSb} .{ }^{20}$ Further increments of the $\mathrm{Sb}$ dose cause a slight redshift in the WL energy.

The different optical transitions appear grouped in shells and can be tentatively labeled in analogy to the twodimensional harmonic-oscillator nomenclature according to their orbital angular momentum quantum number $\left(s\right.$ for $l^{2}=0$, $p$ for $\left.l^{2}=1, \ldots\right)$. This assignment is supported by the fact that, for all samples, the energy difference between the ground state and the first excited state $E_{s}-\mathrm{E}_{p}$ is roughly the same as the difference between the first and the second $E_{p}-\mathrm{E}_{d}$ excited states in good agreement with the harmonic-oscillator approximation. Both $E_{s}-\mathrm{E}_{p}$ and $E_{p}-\mathrm{E}_{d}$ decrease with increasing $\mathrm{Sb}$ dose, whereas, there is a redshift in the ground state. $E_{s}-\mathrm{E}_{p}$ reaches a minimum value of $50 \mathrm{meV}$ for sample $\mathrm{D}, 30 \mathrm{meV}$ smaller than the value measured for the InAs QD reference ensemble. 


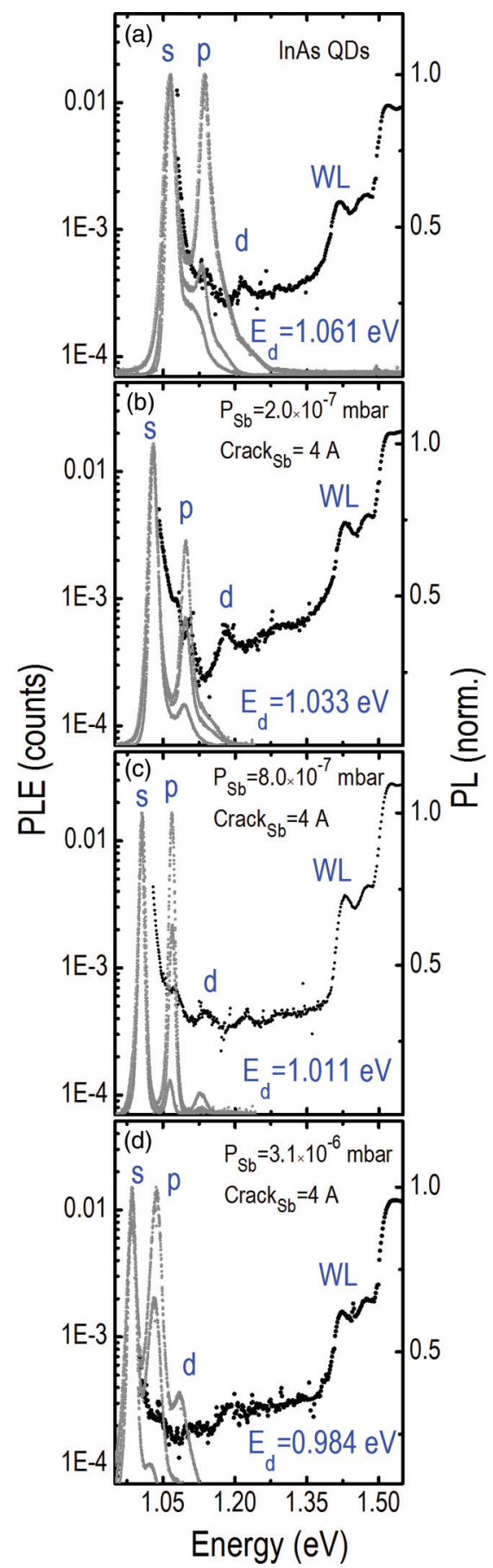

FIG. 1. (Color online) Normalized PL spectra obtained with excitation powers of $0.04,0.4$, and $4 \mathrm{~W} / \mathrm{cm}^{2}$ overlapped with the PLE spectra. The corresponding detection energies are indicated on the right corner of each spectrum. The PL power dependence signal has been measured at $T=20 \mathrm{~K}$, and the PLE powder dependence signal has been measured at $T=16 \mathrm{~K}$.

The modification of the excited-state structure also affects the PL thermal escape activation energies $\left(E_{\mathrm{a}}\right)$. Following the PL-integrated intensity evolution between 20 and $300 \mathrm{~K}$, we have extracted $E_{\mathrm{a}}$ as a function of the Sb dose (Table II). In all cases, a moderate exposure to $\mathrm{Sb}$ before QD capping increases the thermal escape barrier compared with the reference InAs QDs. Sample C, which had the overall best optical properties,
TABLE II. Summary of the PL-integrated intensity, activation energy of the thermal escape process, and decay time of the different samples studied in this paper. PL-integrated intensity values are normalized to the integrated intensity in the reference InAs QD sample.

\begin{tabular}{lcccc}
\hline \hline Sample & \multicolumn{2}{l}{$\begin{array}{l}\text { Integrated intensity } \\
\text { (normalized to InAs QD) }\end{array}$} & & \\
\cline { 2 - 3 } & $T=28 \mathrm{~K}$ & $\mathrm{RT}$ & $E_{\mathrm{a}}(\mathrm{meV})$ & $\tau(\mathrm{ns})$ \\
\hline $\mathrm{A}$ & 1 & 1 & 183 & 0.98 \\
$\mathrm{~B}$ & 1.18 & 5.47 & 218.4 & 1.06 \\
$\mathrm{C}$ & 1.80 & 10.17 & 273.7 & 1.08 \\
$\mathrm{D}$ & 1.07 & 7.85 & 208.6 & 1.53 \\
\hline \hline
\end{tabular}

also shows the biggest increment $\Delta E_{\mathrm{a}} \sim 50 \%$. Further increments of the $\mathrm{Sb}$ dose result in a decrease in $E_{\mathrm{a}}$. This is most likely due to a crossover from hole thermal escape to electron thermal escape with increasing Sb content due to the lower electron affinity of the antimonides, being the thermal escape barrier maximum exactly at the crossover.

\section{MODEL AND DISCUSSION}

To account for the results obtained in PLE, PL versus temperature, and TRPL, the QD electronic structure was calculated for a wide range of QD geometries and compositions. All calculations have been performed with the NEXTNANO ++ package. $^{21}$ The numerical details of the model and material parameters are the same as in Refs. 22 and 23. In this paper, we only summarize the computational results more relevant to our experiments; the full set of results will be reported elsewhere. The reference QDs are modeled as lens shaped and are made of a homogeneous InGaAs alloy $\left(x_{\mathrm{Ga}}=15 \%\right)$. This composition is estimated by comparing our computational results with the ground-state emission energy of the InAs reference sample and remains fixed in all the calculations carried out. Calculations were initially performed in QDs with a homogeneous Sb distribution. However, from the X-STM and TEM analyses reported in Ref. 18, the Sb is definitely not homogeneously distributed. The X-STM revealed that Sb is confined within the QD volume exhibiting a concentration in the apex region twice that at the base. Dark-field 002 TEM images confirmed this, revealing a dark contrast at half-height corresponding to a composition change from Sb-poor (QDbase) to Sb-rich (QD-tip) InGaAsSb alloys. This particular $\mathrm{Sb}$ distribution is considered in the model introducing a different $\mathrm{Sb}$ content in the upper and lower halves of the QDs by describing them as lens splitted at half their height. According to our previously reported AFM measurements, ${ }^{18}$ $\mathrm{Sb}$ exposure causes a QD height increase from 8 to $12 \mathrm{~nm}$ as measured before capping. Nevertheless, this height increase almost completely disappears during capping, and our TEM and X-STM measurements reveal QD heights ranging from 8 to $9 \mathrm{~nm}$ in all cases. Electronic structure calculations performed in order to estimate the effect of the QD height changes have resulted in small shifts in the fundamental transition energy $(\sim 10 \mathrm{meV})$ for QD heights greater than $6 \mathrm{~nm}$. Consequently, the QD height was fixed at $h=8 \mathrm{~nm}$ in all the calculations. Our previous TEM structural characterization also revealed that the 


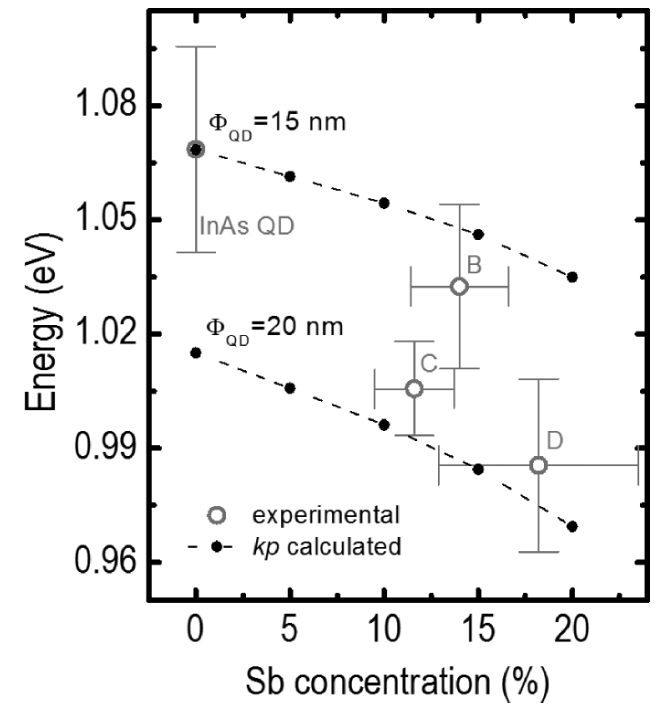

FIG. 2. Photoluminescence energy evolution with Sb concentration in the top half of the QD. The vertical error bars correspond to the PL FWHM, and the horizontal error bars correspond to the uncertainty margin of the $\mathrm{Sb}$ concentration measurements reported in Ref. 18. Results are compared with the $k p$ calculated energy for QDs with two different diameters $\left(F_{\mathrm{QD}}=15\right.$ and $\left.F_{\mathrm{QD}}=20 \mathrm{~nm}\right)$.

QD lateral extent increases with Sb exposure from $15 \pm 3 \mathrm{~nm}$ for InAs QDs to $20 \pm 3 \mathrm{~nm}$ for sample D. The QD lateral size data for all samples are summarized in Table I. We have, thus, performed the calculations for two different QD diameters: 15 and $20 \mathrm{~nm}$.

In the case of the ground-state transition energy for QDs without $\mathrm{Sb}$, the difference between our calculations based on structural characterization of the QDs and our spectroscopy experiments is $66 \mathrm{meV}$. This difference can be accounted for by the fact that the electron-hole Coulomb interaction is not included in the calculations $(\sim 30 \mathrm{meV})$ and various other experimental and computational uncertainties. ${ }^{24}$ All calculated transition energies have been shifted by this $66-\mathrm{meV}$ difference. The results of the calculation are compared with experimental values of the ground-state energy in Fig. 2. The experimentally observed transitions energies can be tentatively explained on the basis of the previously mentioned increased lateral size after Sb incorporation as changes in composition are not enough to cause the observed redshift. Under these considerations, they produce a reasonable fit to the experimentally observed optical transitions. The strong nonlinearity of the composition and emission wavelength changes with the $\mathrm{Sb}$ dose and the fact that we have not been able to produce larger redshifts than those observed for sample D suggest the existence of a solubility limit around 20\% for $\mathrm{Sb}$ at the apex of InAs/GaAs QDs.

In Fig. 3, we show the absorption spectra of a $\mathrm{Ga}_{0.15} \mathrm{In}_{0.85} \mathrm{As} / \mathrm{GaAs}$ QD of 8-nm height and 15-nm diameter (labeled as $\mathrm{Sb} 0 \%$ ). The spectra show the optically active transitions calculated including 14 electron and 14 hole states. The number of states is big enough to contain the first 12 states making up a shell structure of $s, p$, and $d$ states of multiplicity 2,4 , and 6 , respectively. For better comparison with experiments, the calculated spectra have been broadened

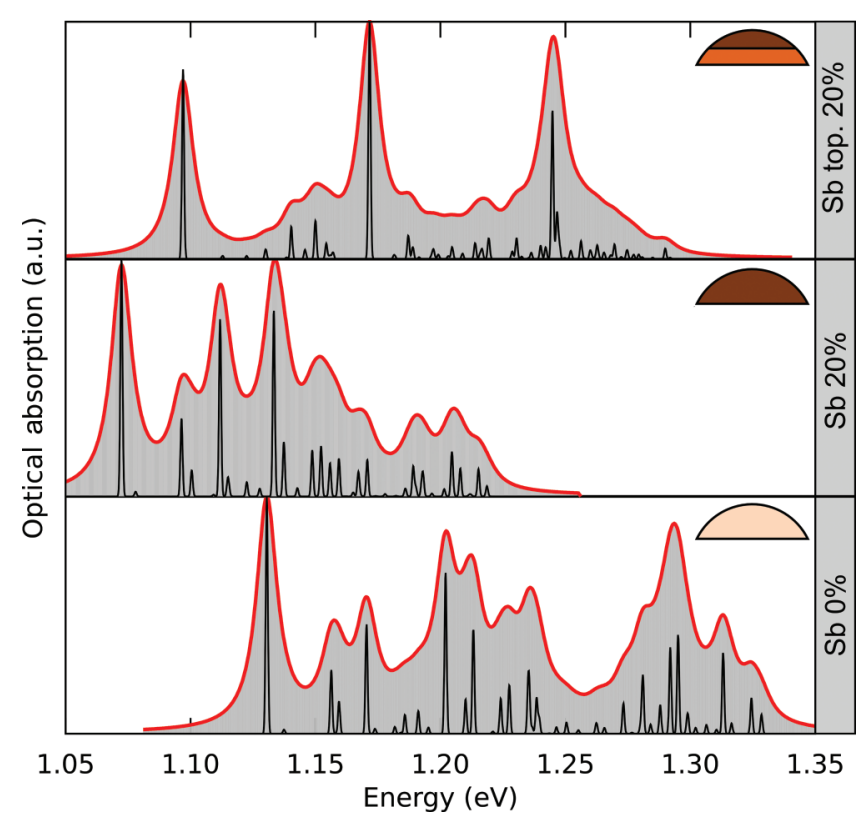

FIG. 3. (Color online) Calculated optical absorption spectra. The spectrum of the bottom panel corresponds to the QD without $\mathrm{Sb}$, the one of the middle panel corresponds to a QD with $20 \%$ Sb distributed homogenously, and finally, that of the top panel corresponds to a QD with $20 \% \mathrm{Sb}$ in the upper half and $10 \%$ in the lower half. For the spectrum in black (red), a homogeneous (inhomogeneous) broadening of 1 (10) $\mathrm{meV}$ is assumed. These results correspond to a QD of 8-nm height and 15-nm diameter. The spectra are computed from 14 electron and 14 hole states.

by convolution with a 10-meV FWHM Gaussian distribution. In high aspect ratio QDs as the ones considered in this study, the selection rules of the optical transitions relax, making possible transitions between states of different shells. In addition, the hole states include contributions from valence bands other than the heavy-hole band, which distorts the simple peak distribution characteristic of the harmonic oscillator even further. As a consequence, the peak structure in QDs of homogeneous composition does not bear a clear resemblance to the spectrum of a simple harmonic oscillator found in our PL spectra [see Fig. 1(c)]. Interestingly such a resemblance is recovered if we assume an inhomogeneous $\mathrm{Sb}$ composition profile inside the QD with a Sb content in the upper half, which is twice the amount found in the lower half. The origin of such particular behavior can be explained by looking at the density of probability of the wave functions. We will come back to this point when discussing Fig. 4. The solutions of the $k p$ model based on the realistic distribution of $\mathrm{Sb}$ in the QDs are in good agreement with both PL and PLE experimental results (Fig. 1). This confirms the strong influence of the Sb-rich top-half QD structure on the InAsSb QDs energy levels described in Ref. 18.

Figure 4 shows the calculated density of the probability of the electron and hole ground-state wave functions. The cross section corresponds to the plane [110] for the same QD geometry as that used in the calculations of Fig. 2. As the concentration of $\mathrm{Sb}$ is higher in the upper than in the bottom half of the QDs, the hole state feels a deeper well towards the apex of the QD. Hence, the wave-function maximum 


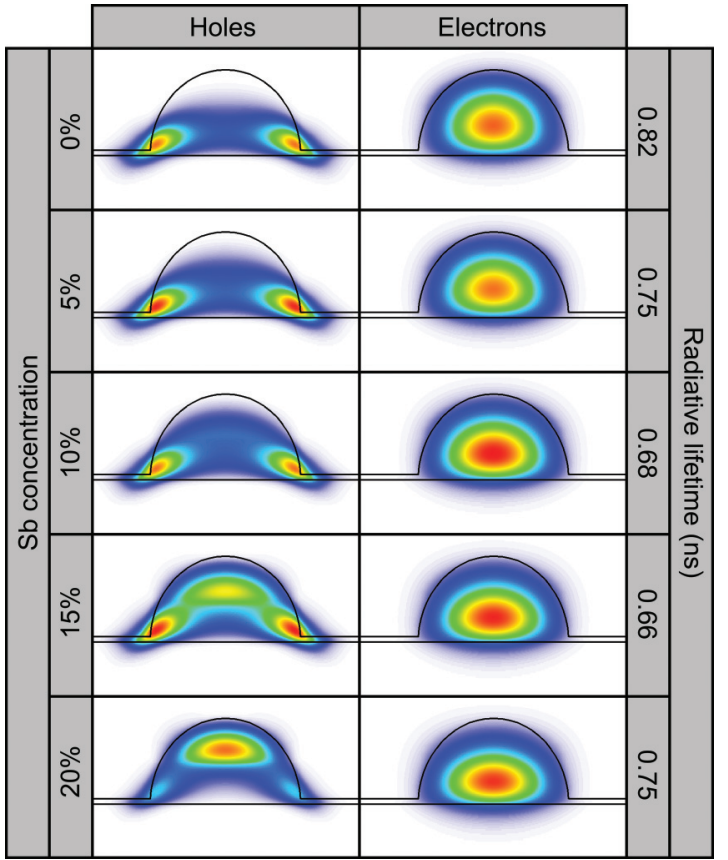

FIG. 4. (Color online) Electron and hole wave functions calculated using a lens-shaped QD. The diameter of the QD is $20 \mathrm{~nm}$. We consider a factor 2 difference in the $\mathrm{Sb}$ concentration between the top and the bottom halves as input.

localization moves from the bottom part of the QDs to its upper half as $\mathrm{Sb}$ concentration increases. This wave-function shift alters the radiative lifetime (see the right-hand side of Fig. 4), which reaches a minimum at a $\mathrm{Sb}$ concentration of $15 \%$ for the upper half, reflecting the enhancement of the electron-hole wave-function overlap. This is consistent with the higher PL-integrated intensity of samples B and C with $14.0 \%$ and $11.6 \% \mathrm{Sb}$ content, respectively. ${ }^{18}$

The correlation of the enhancement of the overlap with that of the PL intensity cannot, however, be directly established in our experiments. Even though the higher the overlap, the higher the optical transition rate, changes in defect densities and carrier dynamics effects seem to play a dominant role in this case. Indeed, the trend of the decay lifetimes collected in Table II does not fit the theoretically predicted trend for the radiative lifetime. The experimental increase in $\tau$ can have its origin in a non-negligible $\mathrm{Sb}$ content in the capping layers and, consequently, a reduction in the nonradiative lifetime due to a shift in the hole wave function. The segregation of $\mathrm{Sb}$ in the barrier might also explain why the evolution of the WL and QDs ground-state energies follows dramatically different trends in our samples [Table I]. Despite Sb exposures causing a redshift in the ground-state energy, they cause a blueshift in the WL absorption edge up to dose $\mathrm{C}$. At higher $\mathrm{Sb}$ doses the WL absorption edge starts to redshift. These considerations depict two clearly separated regimes. At low doses, Sb incorporation is enhanced in the QDs, especially in their apex due to the preferential incorporation of $\mathrm{Sb}$ atoms in the tip of the QDs where strain is minimum. In the low $\mathrm{Sb}$ exposure regime, the InGaAsSb QD optical properties are accurately explained by our model. At higher $\mathrm{Sb}$ exposures, the incorporation limit of $\mathrm{Sb}$ into the QDs is reached but not in the WL. Our theoretical model is less successful in this high $\mathrm{Sb}$ exposure regime as we did not consider the effect of $\mathrm{Sb}$ incorporation into the barrier.

\section{CONCLUSIONS}

The infrared spectra of InAs QDs exposed to Sb immediately before capping have provided a number of clues about how $\mathrm{Sb}$ incorporates and its effects on the electronic structure of the QDs. As the Sb dose is increased, the PL emission redshifts, and the room-temperature intensity increases due to an increased thermal escape activation energy, but the incorporation of $\mathrm{Sb}$ into the QDs tends to saturate, indicating the existence of a solubility limit for this process. Our eight band $k p$ calculations show that the experimentally observed changes in the ground-state energy cannot merely be explained by a composition change but can be explained by considering a change in the QDs' lateral size. Furthermore, the infrared spectra are best reproduced assuming an inhomogeneous $\mathrm{Sb}$ content inside the QDs with a higher Sb content in the upper half of the QDs in agreement with our previous structural characterization results. This characteristic $\mathrm{Sb}$ distribution inside the QDs produces strong changes in the density of states, particularly, in the separation between excitation shells. Incorporation of Sb in InAs QDs shifts the hole wave function to the center of the QD from the edges of the QD where it is otherwise pinned down by the effects of shear stress.

\section{ACKNOWLEDGMENTS}

We acknowledge support by MINECO (Projects No. TEC2011-29120-C05-04 and No. ENE2012-37804-C02-02) and CAM (Projects No. S2009ESP-1503 and No. S2009/ENE1477).
*Present address: Labor für Festkörperphysik HPF F2, Schafmattstrasse 16, $8093 \quad$ Zürich, Switzerland; gonzalez@phys.ethz.ch

${ }^{\dagger}$ Current address: Instituto de Fusion Nuclear, Universidad Politecnica de Madrid, Spain.

${ }^{\ddagger}$ Corresponding author: ripalda@imm.cnm.csic.es

${ }^{1}$ Y. Arakawa and H. Sakaki, Appl. Phys. Lett. 40, 939 (1982).

${ }^{2}$ D. Bimberg, M. Grundmann, and N. N. Ledentsov, Quantum Dot Heterostructures (Wiley, Chichester, 1999).
${ }^{3}$ D. Alonso-Álvarez, A. G. Taboada, J. M. Ripalda, B. Alén, Y. González, L. González, J. M. García, F. Briones, A. Martí, A. Luque, A. M. Sánchez, and S. I. Molina, Appl. Phys. Lett. 93, 123114 (2008).

${ }^{4}$ J. Philips, J. Appl. Phys. 91, 4590 (2002).

${ }^{5}$ M. J. Da Silva, A. A. Quivy, S. Martini, T. E. Lamas, E. C. F. da Silva, and J. R. Leite, J. Cryst. Growth 251, 181 (2003).

${ }^{6}$ H. Liu, T. Wang, Q. Jiang, R. Hogg, F. Tutu, F. Pozzi, and A. Seeds, Nat. Photonics 5, 416 (2011). 
${ }^{7}$ D. Guimmard, M. Ishida, N. Hatori, Y. Nakata, H. Sudo, T. Yamamoto, H. Sugawara, and Y. Arakawa, IEEE Photonics Technol. Lett. 20, 10 (2008)

${ }^{8}$ T. Yang, J. Tatebayashi, S. Tsukamoto, M. Nishioka, and Y. Arakawa, Appl. Phys. Lett. 84, 2817 (2004).

${ }^{9}$ K. Yamaguchi, T. Kaizu, K. Yujobo, and Y. Saito, J. Cryst. Growth 237-239, 1301 (2002).

${ }^{10}$ K. Akahane, N. Yamamoto, and N. Ohtani, Physica E (Amsterdam) 21, 295 (2004).

${ }^{11}$ Y. Liu, M. J. Steer, T. J. Badcock, D. J. Mowbray, M. S. Skolnick, P. Navaretti, K. M. Groom, M. Hopkinson, and R. A. Hogg, Appl. Phys. Lett. 86, 143108 (2005).

${ }^{12}$ J. M. Ripalda, D. Granados, Y. González, A. M. Sánchez, S. I. Molina, and J. M. García, Appl. Phys. Lett. 87, 202108 (2005).

${ }^{13}$ H. Y. Liu, M. J. Steer, T. J. Badcock, D. J. Mowbray, M. S. Skolnick, F. Suarez, J. S. Ng, M. Hopkinson, and J. P. R. David, J. Appl. Phys. 99, 046104 (2006).

${ }^{14}$ J. M. Ulloa, R. Gargallo-Caballero, M. Bozkurt, M. del Moral, A. Guzmán, P. M. Koenraad, and A. Hierro, Phys. Rev. B 81, 165305 (2010).

${ }^{15}$ J. M. Ulloa, J. M. Llorens, B. Alén, D. F. Reyes, D. L. Sales, D. González, and A. Hierro, Appl. Phys. Lett. 101, 253112 (2012).
${ }^{16}$ J. He, C. J. Reyner, B. L. Liang, K. Nunna, D. L. Huffaker, N. Pavarelli, K. Gradkowski, T. J. Ochalski, G. Huyet, V. G. Dorogan, Y. I. Mazur, and G. J. Salamo, Nano Lett. 10, 3052 (2010).

${ }^{17}$ Yu. I. Mazur, V. G. Dorogan, G. J. Salamo, G. G. Tarasov, B. L. Liang, C. J. Reyner, K. Nunna, and D. L. Huffaker, Appl. Phys. Lett. 100, 033102 (2012).

${ }^{18}$ A. G. Taboada, A. M. Sánchez, A. M. Beltrán, M. Bozkurt, D. Alonso-Álvarez, B. Alén, A. Rivera, J. M. Ripalda, J. M. Llorens, J. Martín-Sánchez, Y. González, J. M. Ulloa, J. M. García, S. I. Molina, and P. M. Koenraad, Phys. Rev. B 82, 235316 (2010).

${ }^{19}$ E. H. Bogarbus and H. B. Bebb, Phys. Rev. 176, 993 (1968).

${ }^{20} \mathrm{M}$. Yano, H. Yokose, Y. Iwai, and M. Inoue, J. Cryst. Growth 111, 609 (1991).

${ }^{21}$ S. Birner, T. Zibold, T. Andlauer, T. Kubis, M. Sabathil, A. Trellakis, and P. Vogl, IEEE Trans. Electron Devices 54, 2137 (2007); [http://www.nextnano.de]

${ }^{22}$ J. M. Ulloa, J. M. Llorens, M. del Moral, M. Bozkurt, P. M. Koenraad, and A. Hierro, J. Appl. Phys. 112, 074311 (2012).

${ }^{23}$ J. M. Llorens, A. G. Taboada, J. M. Ripalda, D. Alonso-Álvarez, B. Alén, J. Martín-Sánchez, J. M. García, Y. González, A. M. Sánchez, A. M. Beltrán, P. L. Galindo, and S. I. Molina, J. Phys.: Conf. Ser. 245, 012081 (2010).

${ }^{24}$ P. Klenovsky, V. Krapek, D. Munzar, and J. Humlicek, Appl. Phys. Lett. 97, 203107 (2010). 